\title{
On degeneracy of dispersive waves at the bulk wave velocities
}

\author{
Alla Ilyashenko $^{1^{*}}$ and Sergey Kuznetsov ${ }^{1,2,3}$ \\ ${ }^{1}$ Moscow State University of Civil Engineering, Yaroslavsckoe sh. 26, Moscow, Russia \\ ${ }^{2}$ Ishlinsky Institute for Problems in Mechanics RAS, Pr. Vernadskogo 101, Moscow, Russia \\ ${ }^{3}$ Bauman Moscow State Technical University, $2^{\text {nd }}$ Institutskaya 5, Moscow, Russia
}

\begin{abstract}
Degeneracy of the linear dispersion wave equation at the phase velocities coinciding with the bulk wave velocities is observed and analysed. Spectral analysis of Pochhammer - Chree equation is performed. The corrected analytical solutions for components of the displacement fields are constructed, accounting degeneracy of the secular equations and the corresponding solutions.
\end{abstract}

\section{Introduction}

The paper is devoted to analyzing and correcting solutions of the Pochhammer - Chree wave equation at phase velocities coinciding with the longitudinal $\left(c_{1}\right)$ and shear $\left(c_{2}\right)$ bulk wave velocities, at which degeneracy of the Bessel equation occurs. The corrected dispersion equations at these phase velocities are constructed, revealing peculiarities in polarization of the corresponding Pochhammer - Chree longitudinal waves.

Since the first derivation of the Pochhammer - Chree equation for harmonic waves propagating in a cylindrical rod [1 - 3] and numerous subsequent works [4 - 14] it was assumed that the solution of the dynamic equations for harmonic waves in a circular rod reduces to the Bessel equations regardless of the phase velocity.

However, as it will be shown later on, at the phase velocities coinciding with $c_{1}$ and $c_{2}$ bulk wave velocities, the corresponding dynamic equations do not lead to Bessel equations, and hence, the solutions for the dynamic equations and the dispersion equations should be reworked. These solutions will be constructed and analyzed below.

\section{Principle equations}

Equation of motion for an isotropic medium at absence of body forces can be represented in a form

$$
c_{1}^{2} \nabla \operatorname{div} \mathbf{u}-c_{2}^{2} \operatorname{rot} \operatorname{rot} \mathbf{u}=\partial_{t t}^{2} \mathbf{u},
$$

where $\mathbf{u}$ is the displacement field, $c_{1}, c_{2}$ are velocities of bulk longitudinal and shear waves respectively:

*Corresponding author: avi_56@mail.ru 


$$
c_{1}=\sqrt{\frac{\lambda+2 \mu}{\rho}}, \quad c_{2}=\sqrt{\frac{\mu}{\rho}} .
$$

In (2.2) $\lambda, \mu$ are Lame's constants, and $\rho$ is a material density.

The Helmholtz representation for the displacement field $\mathbf{u}$ yields

$$
\mathbf{u}=\nabla \Phi+\operatorname{rot} \Psi
$$

where $\Phi$ and $\Psi$ are scalar and vector potentials respectively. In cylindrical coordinates representation (2.3) for the physical components of the displacement field, becomes

$$
\begin{aligned}
& u_{r}=\frac{\partial \Phi}{\partial r}+\frac{1}{r} \frac{\partial \Psi_{z}}{\partial \theta}-\frac{\partial \Psi_{\theta}}{\partial z} \\
& u_{\theta}=\frac{1}{r} \frac{\partial \Phi}{\partial \theta}+\frac{\partial \Psi_{r}}{\partial z}-\frac{\partial \Psi_{z}}{\partial r} \\
& u_{z}=\frac{\partial \Phi}{\partial z}+\frac{1}{r} \frac{\partial}{\partial r}\left(r \Psi_{\theta}\right)-\frac{1}{r} \frac{\partial \Psi_{r}}{\partial \theta}
\end{aligned}
$$

In (2.4) it is assumed that coordinate $z$ directs along central axis of the rod. It is also assumed that the displacement field is axially symmetric, that yields

$$
u_{\theta}=0 \text {. }
$$

Substituting representation (2.3) into equation of motion (2.1) yields

$$
c_{1}^{2} \Delta \Phi=\ddot{\Phi}, \quad c_{2}^{2} \Delta \Psi=\ddot{\Psi} .
$$

For a plane harmonic wave propagating along axis $z$, potentials (2.6) can be represented in a form

$$
\Phi=\Phi_{0}\left(\boldsymbol{x}^{\prime}\right) e^{i \gamma(z-c t)}, \quad \Psi=\Psi_{0}\left(\boldsymbol{x}^{\prime}\right) e^{i \gamma(z-c t)},
$$

where, as before, $\gamma$ is the wave number related to the phase speed $c$ and circular frequency $\omega$ by equation

$$
\gamma=\frac{\omega}{c}
$$

In (2.7) $\boldsymbol{x}^{\prime}$ is the (vector) coordinate in the cross section of a $\operatorname{rod}\left(\mathbf{x}^{\prime}=\mathbf{x}-(\mathbf{n} \cdot \mathbf{x}) \mathbf{n}\right)$, where $\mathbf{n}$ is the wave vector; and $z=\mathbf{n} \cdot \mathbf{x}$.

Substituting representations (2.7) into Eqs. (2.6), yields Helmholtz equations for the potentials

$$
\Delta \Phi_{0}+\left(\frac{c^{2}}{c_{1}^{2}}-1\right) \gamma^{2} \Phi_{0}=0, \quad \Delta \Psi_{0}+\left(\frac{c^{2}}{c_{2}^{2}}-1\right) \gamma^{2} \Psi_{0}=0 .
$$

Axial symmetry of $\Phi_{0}$ ensures [4]

$$
\frac{\partial \Phi_{0}}{\partial \theta}=0
$$

\subsection{Non-degenerate case $c \neq c_{1} \vee c_{2}$}

If $c \neq c_{1} \vee c_{2}$ ( $c$ is phase velocity), then Eqs. (2.9) result in Bessel equation for $\Phi_{0}$ and $\Psi_{0}$ potentials. The corresponding solution for the scalar potential has the form

$$
\Phi_{0}(r)=C_{1} J_{0}\left(q_{1} r\right)+C_{2} Y_{0}\left(q_{1} r\right)
$$

and for the vector potential the corresponding solutions become 


$$
\begin{aligned}
& \Psi_{\theta}(r)=C_{3} J_{1}\left(q_{2} r\right)+C_{4} Y_{1}\left(q_{2} r\right) \\
& \Psi_{r}(r)=C_{5} J_{1}\left(q_{2} r\right)+C_{6} Y_{1}\left(q_{2} r\right), \\
& \Psi_{z}(r)=C_{7} J_{0}\left(q_{2} r\right)+C_{8} Y_{0}\left(q_{2} r\right)
\end{aligned}
$$

Herein, $C_{k}, k=1, \ldots, 8$ are unknown (complex) coefficients, and

$$
q_{n}^{2}=\left(\frac{c^{2}}{c_{n}^{2}}-1\right) \gamma^{2}, \quad n=1,2 .
$$

Axial symmetry of potential $\Psi_{0}$ is satisfied by the following equations [4]

$$
\frac{\partial \Psi_{r}}{\partial \theta}=\frac{\partial \Psi_{\theta}}{\partial \theta}=\frac{\partial \Psi_{z}}{\partial \theta}=0
$$

Axial symmetry of the vector potential $\Psi$ imposes another restriction [4]:

$$
\Psi_{r}=\Psi_{z}=0 \text {. }
$$

Now, accounting (2.4), (2.5) (2.12), (2.15), the desired vector field corresponding to the propagating longitudinal axially symmetric harmonic wave, becomes [7]:

$$
\begin{aligned}
& u_{r}=-\left[q_{1}\left(C_{1} J_{1}\left(q_{1} r\right)+C_{2} Y_{1}\left(q_{1} r\right)\right)+i \gamma\left(C_{3} J_{1}\left(q_{2} r\right)+C_{4} Y_{1}\left(q_{2} r\right)\right)\right] e^{i \gamma(z-c t)} \\
& u_{\theta}=0 \\
& u_{z}=\left[i \gamma\left(C_{1} J_{0}\left(q_{1} r\right)+C_{2} Y_{0}\left(q_{1} r\right)\right)+q_{2}\left(C_{3} J_{0}\left(q_{2} r\right)+C_{4} Y_{0}\left(q_{2} r\right)\right)\right] e^{i \gamma(z-c t)}
\end{aligned}
$$

Since components (2.16) vector field should be finite at $r=0$ and noting that at $r \rightarrow 0$ Bessel's functions $Y_{0}, Y_{1}$ are unbounded, the final representation flows out from (2.16)

$$
\begin{aligned}
& u_{r}=-\left[q_{1} C_{1} J_{1}\left(q_{1} r\right)+i \gamma C_{3} J_{1}\left(q_{2} r\right)\right] e^{i \gamma(z-c t)} \\
& u_{\theta}=0 \\
& u_{z}=\left[i \gamma C_{1} J_{0}\left(q_{1} r\right)+q_{2} C_{3} J_{0}\left(q_{2} r\right)\right] e^{i \gamma(z-c t)}
\end{aligned}
$$

\subsection{Degenerate case, $c=c_{1}$}

At $c=c_{1}$ Eqs. (2.9) become

$$
\Delta \Phi_{0}=0, \quad \Delta \Psi_{0}+\left(\frac{c_{1}^{2}}{c_{2}^{2}}-1\right) \gamma^{2} \Psi_{0}=0
$$

Now, the first Eq. (2.18) yields

$$
\Phi_{0}(r)=C_{1}+C_{2} \ln r
$$

Taking into account conditions (2.14), (2.15), and solutions (2.19), (2.12), the desired components of the axially symmetric displacement field become

$$
\begin{aligned}
& u_{r}=\left[r^{-1} C_{2}-i \gamma\left(C_{3} J_{1}\left(q_{2} r\right)+C_{4} Y_{1}\left(q_{2} r\right)\right)\right] e^{i \gamma(z-c t)} \\
& u_{\theta}=0 \\
& u_{z}=\left[i \gamma\left(C_{1}+C_{2} \ln r\right)+q_{2}\left(C_{3} J_{0}\left(q_{2} r\right)+C_{4} Y_{0}\left(q_{2} r\right)\right)\right] e^{i \gamma(z-c t)} .
\end{aligned}
$$

And accounting that for a solid cylinder the corresponding displacement fields should be finite at $r=0$, Eqs. (2.20) by taking $C_{2}=0$ transform into 


$$
\begin{aligned}
& u_{r}=-i \gamma C_{3} J_{1}\left(q_{2} r\right) e^{i \gamma(z-c t)} \\
& u_{\theta}=0 \\
& u_{z}=i \gamma C_{1}+q_{2} C_{3} J_{0}\left(q_{2} r\right) e^{i \gamma(z-c t)}
\end{aligned}
$$

\subsection{Degenerate case, $c=c_{2}$}

At $c=c_{2}$ Eqs. (2.9) become

$$
\Delta \Phi_{0}+\left(\frac{c_{2}^{2}}{c_{1}^{2}}-1\right) \gamma^{2} \Phi_{0}=0, \quad \Delta \Psi_{0}=0 .
$$

That in view of conditions (2.14), (2.15) yields the following solution for component $\Psi_{\theta}(r)$

$$
\Psi_{\theta}(r)=C_{3} r+C_{4} r^{-1}
$$

Taking into account Eqs. (2.4), components of the required displacement field become

$$
\begin{aligned}
& u_{r}=-\left[q_{1}\left(C_{1} J_{1}\left(q_{1} r\right)+C_{2} Y_{1}\left(q_{1} r\right)\right)-i \gamma\left(C_{3} r+C_{4} r^{-1}\right)\right] e^{i \gamma(z-c t)} \\
& u_{\theta}=0 \\
& u_{z}=\left[i \gamma\left(C_{1} J_{0}\left(q_{1} r\right)+C_{2} Y_{0}\left(q_{1} r\right)\right)+2 C_{3}\right] e^{i \gamma(z-c t)}
\end{aligned}
$$

And accounting that for a solid cylinder the corresponding displacement field should be finite at $r=0$, results in

$$
\begin{aligned}
& u_{r}=-\left[q_{1} C_{1} J_{1}\left(q_{1} r\right)-i \gamma C_{3} r\right] e^{i \gamma(z-c t)} \\
& u_{\theta}=0 \\
& u_{z}=i\left[\gamma C_{1} J_{0}\left(q_{1} r\right)+2 C_{3}\right] e^{i \gamma(z-c t)}
\end{aligned}
$$

Remark 2.1. It should be noted that the similar degeneracy occurs for Lamb waves in a plate at the phase velocity $c$ coinciding with $c_{1}$ and $c_{2}$ velocities. For example, at $c=c_{2}$ one of components of the vector potential reduces to

$$
\psi\left(x^{\prime}\right)=C_{1}+C_{2} x^{\prime}
$$

instead of solution in hyperbolic functions at $c \neq c_{2}[11]$ :

$$
\psi\left(x^{\prime}\right)=C_{1} \sinh \left(\gamma x^{\prime}\right)+C_{2} \cosh \left(\gamma x^{\prime}\right)
$$

where the multiplier $\gamma$ is defined by the following expression $\gamma=\left(1-c^{2} / c_{2}^{2}\right)^{1 / 2}$, see [11].

\section{Degenerate dispersion equations at $c_{1}, c_{2}$ phase velocities}

The traction-free boundary conditions on a lateral cylindrical surface at $r=R$ have the form

$$
\left.\mathbf{t}_{\mathbf{v}} \equiv(\lambda(\operatorname{tr} \boldsymbol{\varepsilon}) \mathbf{v}+2 \mu \varepsilon \cdot \mathbf{v})\right|_{r=R}=0
$$

where $\boldsymbol{v}$ is the (outward) surface normal. 


\subsection{Dispersion equation at non-degenerate case $c \neq c_{1} \vee c_{2}$}

Substituting displacement components (2.17) into boundary conditions (3.1), yields the following equations written up to the exponential multiplier $e^{i \gamma(z-c t)}$

$$
\begin{gathered}
t_{r r} \equiv \lambda I_{\varepsilon}+2 \mu \varepsilon_{r r}=-\left[\begin{array}{l}
\lambda\left(q_{1}^{2}+\gamma^{2}\right) J_{0}\left(q_{1} r\right) C_{1}+ \\
\left.+\frac{2 \mu}{r}\left[\begin{array}{l}
q_{1} C_{1}\left(q_{1} r J_{0}\left(q_{1} r\right)-J_{1}\left(q_{1} r\right)\right)+ \\
+i \gamma C_{3}\left(q_{2} r J_{0}\left(q_{2} r\right)-J_{1}\left(q_{2} r\right)\right)
\end{array}\right]\right]_{r=R} .
\end{array}=0\right. \\
\left.t_{r z} \equiv 2 \mu \varepsilon_{r z}=-\mu\left[\begin{array}{l}
i \gamma\left[q_{1} C_{1} J_{1}\left(q_{1} r\right)+i \gamma C_{3} J_{1}\left(q_{2} r\right)\right] \\
+\left[i \gamma q_{1} C_{1} J_{1}\left(q_{1} r\right)+q_{2}^{2} C_{3} J_{1}\left(q_{2} r\right)\right]
\end{array}\right]\right]_{r=R}=0
\end{gathered}
$$

Equations (3.2) can be rewritten in a matrix form

$$
\mathbf{A} \cdot \vec{C}=0, \quad \vec{C}=\left(\begin{array}{l}
C_{1} \\
C_{3}
\end{array}\right),
$$

where $\mathbf{A}$ is a square and non-symmetric $2 \times 2$ matrix with complex coefficients:

$$
\begin{aligned}
& A_{11}=-\left(\left(q_{1}^{2}+\gamma^{2}\right) \frac{c_{1}^{2}}{c_{2}^{2}}-2 \gamma^{2}\right) J_{0}\left(q_{1} R\right)+\frac{2 q_{1}}{R} J_{1}\left(q_{1} R\right) \\
& A_{12}=-\frac{2 i \gamma}{R}\left(q_{2} R J_{0}\left(q_{2} R\right)-J_{1}\left(q_{2} R\right)\right) \\
& A_{21}=-2 i \gamma q_{1} J_{1}\left(q_{1} R\right) \\
& A_{22}=-\left(q_{2}^{2}-\gamma^{2}\right) J_{1}\left(q_{2} R\right)
\end{aligned}
$$

At deriving (3.4) from (3.2) the following identity was used

$$
\frac{\lambda}{\mu}=\frac{c_{1}^{2}}{c_{2}^{2}}-2 .
$$

Now the desired dispersion equation can be represented in the form

$$
\operatorname{det} \mathbf{A}=0 \text {. }
$$

Two-dimensional (right) eigenvectors related to vanishing eigenvalues (kernel eigenvectors) of matrix $\mathbf{A}$ define polarization of the corresponding Pochhammer - Chree waves.

Substituting components (3.4) into Eq. (3.6) yields the dispersion equation in the form [7]

$$
\begin{aligned}
& 4 \gamma^{2} q_{1} q_{2} J_{0}\left(q_{2} R\right) J_{1}\left(q_{1} R\right)-\frac{2 q_{1}}{R}\left(q_{2}^{2}+\gamma^{2}\right) J_{1}\left(q_{1} R\right) J_{1}\left(q_{2} R\right)+ \\
& +\left(q_{2}^{2}-\gamma^{2}\right)\left(\left(q_{1}^{2}+\gamma^{2}\right) \frac{c_{1}^{2}}{c_{2}^{2}}-2 \gamma^{2}\right) J_{0}\left(q_{1} R\right) J_{1}\left(q_{2} R\right)=0
\end{aligned}
$$

\subsection{Dispersion equation at degenerate case $c=c_{1}$}

In view of Eqs. (2.21), the surface traction components at $r=R$ become 


$$
\begin{aligned}
& t_{r r} \equiv \lambda I_{\varepsilon}+2 \mu \varepsilon_{r r}=-2 \mu\left[i \gamma\left(q_{2} J_{0}\left(q_{2} r\right)-r^{-1} J_{1}\left(q_{2} r\right)\right) C_{3}\right]_{r=R}=0 \\
& t_{r z} \equiv 2 \mu \varepsilon_{r z}=-\mu\left[\left(q_{2}^{2}-\gamma^{2}\right) J_{1}\left(q_{2} r\right) C_{3}\right]_{r=R}=0
\end{aligned}
$$

Suppose initially that $\omega \neq 0$, then direct analysis of Eq. $(3.8)_{2}$ reveals that either $J_{1}\left(q_{2} R\right)=0$ or $q_{2}^{2}-\gamma^{2}=0$. The first option is inconsistent with Eq. (3.8) $)_{1}$, as Bessel functions $J_{0}$ and $J_{1}$ have no common roots. The second option in view of (2.13) means

$$
c_{1}^{2}=2 c_{2}^{2}
$$

or $q_{2}=1$. Note, that the case $\omega=0$ at this stage is not considered. But, condition (3.9) means a very specific material with vanishing Poisson's ratio, that flows out from analyzing expressions (2.2). Now, assuming that (3.9) takes place, Eq. (3.8) $)_{1}$ yields the following equation

$$
J_{0}(R)-R^{-1} J_{1}(R)
$$

which satisfies at some specific values of the radius $R$ independently of $\omega \neq 0$. Thus, the case (3.9) at $\omega \neq 0$ does not lead to any meaningful dispersion relation.

Suppose now that $\omega=0$, then direct analysis of Eqs. (3.8) reveals that in such a case both equations are satisfied identically, ensuring that a pair

$$
\left(\omega=0 ; c=c_{1}\right)
$$

satisfies the dispersion relation and that is the unique solution irrelevant of physical properties. Thus, at $c=c_{1}$ all the dispersion curves vanish.

\subsection{Dispersion equation at degenerate case $c=c_{2}$}

In view of Eqs. (2.25), the surface traction components at $r=R$ become

$$
\begin{aligned}
& t_{r r} \equiv \lambda I_{\varepsilon}+2 \mu \varepsilon_{r r}=-\left[\begin{array}{l}
\lambda\left[\left(q_{1}^{2}+\gamma^{2}\right) J_{0}\left(q_{1} r\right) C_{1}-2 \gamma(i-1) C_{3}\right] \\
+2 \mu\left[q_{1}\left(q_{1} J_{0}\left(q_{1} r\right)-r^{-1} J_{1}\left(q_{1} r\right)\right) C_{1}-i \gamma C_{3}\right]
\end{array}\right]_{r=R}=0 \\
& t_{r z} \equiv 2 \mu \varepsilon_{r z}=-\mu\left[i \gamma\left[q_{1} C_{1} J_{1}\left(q_{1} r\right)-i \gamma C_{3} r\right]+i \gamma q_{1} C_{1} J_{1}\left(q_{1} r\right)\right]_{r=R}=0
\end{aligned}
$$

And, the corresponding matrix dispersion equation takes form (3.6), or if expanded, it becomes

$$
R\left(2 \gamma^{2}-\frac{c_{1}^{2}}{c_{2}^{3}}\left(q_{1}^{2}+\gamma^{2}\right)\right) J_{0}\left(q_{1} R\right)+2 q_{1}\left((3+4 i)-2 \frac{c_{1}^{2}}{c_{2}^{3}}(i+1)\right) J_{1}\left(q_{1} R\right)=0
$$

Presumably, the most interesting is disappearing all the terms not containing Bessel functions in the resulting Eq. (3.13).

\section{Conclusions}

The exact solutions of the linear Pochhammer - Chree equation for propagating harmonic axisymmetric longitudinal waves $L(0, m)$ in a cylindrical body, were analyzed, revealing that at the phase velocities coinciding with the bulk wave velocities $c_{1}$ and $c_{2}$, the Pochhammer - Chree equation becomes degenerate leading to solutions involving functions other than Bessel. 
At these phase velocities the correct dispersion equations were constructed and analyzed; see Eqs. (3.8) and (3.13). It was observed that at $c=c_{1}$ the corresponding dispersion equation has no solutions except a trivial pair $\left(\omega=0 ; c=c_{1}\right)$, while at $c=c_{2}$ the corrected dispersion equation contains non-trivial solutions.

Spectral analysis of the correct dispersion equation at $c=c_{2}$ enabled constructing analytical expressions for components of the displacement field; see Eqs. (4.11) and (4.12). It should also be noted that the similar degeneracy at the bulk wave velocities occurs for propagating Lamb and SH waves; see [15 - 16].

The authors thank the Russian Foundation for Basic Research Grants 17-08-00311; 18-58-41001 and (SVK) the RFBR Grant 19-01-00100 along with the Programs 27, 32, 55 of the Presidium of RAS for partial financial support.

\section{References}

1. Pochhammer L 1876 Ueber die Fortpflanzungsgeschwindigkeiten kleiner Schwingungen in einem unbegrenzten isotropen Kreiscylinder J. Reine Angew. Math. 81 324-36

2. Chree C 1886 Longitudinal vibrations of a circular bar Quart. J. Pure Appl. Math. 21 287-98

3. Chree C 1889 The equations of an isotropic elastic solid in polar and cylindrical coordinates, their solutions and applications Trans. Cambridge Philos. Soc. 14 250-309

4. Mindlin R D and McNiven H D 1960 Axially symmetric waves in elastic rods Trans. ASME. J. Appl. Mech. 27 145-51

5. Meeker T R and Meitzler A H 1964 Guided wave propagation in elongated cylinders and plates In: Physical acoustics. Principles and methods. Acad. Press. N.Y., vol. 1A., pp. 111-67

6. Kolsky H 1964 Stress waves in solids J. Sound Vibr. 1 88-110

7. Zemanek J 1972 An experimental and theoretical investigation of elastic wave propagation in a cylinder J. Acoust. Soc. Amer. $51265-83$

8. Shawagfeh N and Kaya D 2004 Series solution to the Pochhammer-Chree equation and comparison with exact solutions Computers and Mathematics with Applications 47 1915-20

9. Valsamos G, Casadei F and Solomos G 2013 A numerical study of wave dispersion curves in cylindrical rods with circular cross-section Applied and Computational Mechanics 7 99-114

10. Ilyashenko A V and Kuznetsov S V 2018 Pochhammer-Chree waves: polarization of the axially symmetric modes Arch. Appl. Mech. 88 1385-94

11. Ilyashenko A V and Kuznetsov S V 2018 Longitudinal Pochhammer - Chree waves in mild auxetics and non-auxetics J. Mech. 34 1-8

12. Ilyashenko A V and Kuznetsov S V 2018 Horizontally polarized shear waves in stratified anisotropic (monoclinic) media Arch. Mech. 70 305-15

13. Kuznetsov S V 2018 Pochhammer-Chree waves in rods: degeneracy at the bulk wave velocities Zeitschrift für angewandte Mathematik und Physik 69 142-8

14. Kuznetsov S V 2018 Abnormality of the longitudinal Pochhammer-Chree waves in the vicinity of C2 phase speed JVC/Journal of Vibration and Control 24 5642-9

15. Kuznetsov S V 2014 Lamb waves in anisotropic plates (review) Acoustical Physics 60, 95-103

16. Djeran-Maigre I and Kuznetsov S V 2008 Solitary SH waves in two-layered tractionfree plates Comptes Rendus. Mécanique 336 102-7 